\title{
The Effect of COVID-19 Pandemic and Social Restrictions on Depression Rates and Maternal Attachment in Immediate Postpartum Women: a Preliminary Study
}

\author{
Z. Asli Oskovi-Kaplan ${ }^{1} \cdot$ Gül Nihal Buyuk ${ }^{1} \cdot$ A. Seval Ozgu-Erdinc ${ }^{1} \cdot$ H. Levent Keskin ${ }^{1}$ • \\ Alper Ozbas ${ }^{1} \cdot$ Ozlem Moraloglu Tekin ${ }^{1}$
}

Accepted: 30 August 2020/ Published online: 4 September 2020

(C) Springer Science+Business Media, LLC, part of Springer Nature 2020

\begin{abstract}
We aimed to evaluate the postpartum depression rates and maternal-infant bonding status among immediate postpartum women, whose last trimester overlapped with the lockdowns and who gave birth in a tertiary care center which had strong hospital restrictions due to serving also for COVID-19 patients, in the capital of Turkey. The low-risk term pregnant women who gave birth were given the surveys Edinburgh Postpartum Depression Scale (EPDS) and Maternal Attachment Inventory (MAI) within $48 \mathrm{~h}$ after birth. A total of 223 women were recruited. The median score obtained from the EPDS was 7 (7) and $33(14.7 \%)$ of the women were determined to have a risk for postpartum depression. The median scores of the EPDS inventory of depressive women were 15 (3). The median MAI score of 223 women was 100 (26); and the MAI scores of women with depression were significantly lower than the controls [73 (39) vs. 101 (18) respectively, $\boldsymbol{p}<\mathbf{0 . 0 0 1}$ ]. Evaluation of the factors that affect the psychological status of pregnant and postpartum women will lead the healthcare system to improve the implementations during the COVID-19 pandemic.
\end{abstract}

Keywords Pregnancy $\cdot$ Postpartum depression $\cdot$ Maternal bonding $\cdot$ COVID-19 $\cdot$ Pandemic

\section{Introduction}

The coronavirus (COVID-19) spread out from China and caused a pandemic [1]. The number of cases gradually increased and caused governments to take precautions in every area to

Z. Asli Oskovi-Kaplan aslioskovi@gmail.com

1 Department of Obstetrics and Gynecology, Ministry of Health Ankara City Hospital, Maternity Hospital, Bilkent, Ankara, Turkey 
control the number of newly infected cases. Social restrictions and lockdowns were carried out in many countries.

The first case of Turkey was reported on 11 March 2020 and the Government and the Ministry of Health took immediate action for social restrictions [2]. The schools closed and online education started, many workplaces implemented home-office working, the social areas such as parks, shopping malls, many shops, cinemas, cafes, and restaurants were closed; traveling across the cities were prohibited, the flights were canceled and weekend lock-downs were proclaimed between the March - June 2020. By 01 June 2020, there were 164,769 infected cases and a total of 4563 deaths in Turkey (https://covid19.saglik.gov.tr/).

In the beginning months of the outbreak, so less was known about the prognosis of COVID-19 infection during pregnancy, the risk of vertical transmission, and the management as well as there was very limited knowledge about the management and treatment of COVID19 infection in general. Pregnant women and neonates are known to be more vulnerable to several respiratory infections such as influenza when compared with the health non-pregnant population according to previous pandemic outcome reports [3, 4].

The quarantine during pandemic had psychological consequences such as increased posttraumatic stress symptoms, confusion and anger which were associated with financial status, fear of infection, boredom, inadequate reach for supplies, inadequate information, and quarantine duration $[5,6]$. Only limited studies have been performed on the psychosocial status of pregnant and postpartum women during the COVID-19 pandemic lockdown. Most of the maternity wards have implemented restrictions during the pandemic. A concern on the pregnant women's psychological status was previously reported, considering the restrictions such as not allowing the pregnant woman's partner during delivery or prohibiting postpartum hospital visits, which would make it more difficult to cope for vulnerable postpartum mothers $[7,8]$. Furthermore, concerns on the effect of maternal stress during pregnancy may cause neurodevelopmental disorders on offspring have been stated [9]. Therefore in the current study, we aimed to evaluate the postpartum depression rates and maternal-infant bonding status among immediate postpartum women, whose last trimester overlapped with the lockdowns and who gave birth in a tertiary care center which had strong hospital restrictions due to serving also for COVID-19 patients, in the capital of Turkey.

\section{Material-Method}

The study was conducted in the obstetrics department of a tertiary center, which was also a COVID-19 pandemic referral hospital in Ankara, Turkey after an ethical approval was obtained.

The survey was conducted in June 2020. The low-risk term pregnant women who gave birth were given the surveys Edinburgh Postpartum Depression Scale and Maternal Attachment Inventory within $48 \mathrm{~h}$ after birth [10-13]. Women with high-risk pregnancies (preterm delivery, intrauterine growth restriction, fetal anomalies, multiple pregnancies, etc.), diagnosed with chronic diseases (autoimmune, neurologic, cardiac, renal diseases, etc.), women previously diagnosed with a psychiatric disease, women who had COVID-19 disease during pregnancy or a relative diagnosed with COVID-19 disease were excluded from the study.

A total of 525 women gave birth during the study period (Fig. 1). From the total, 220 women did not accept to participate or gave inadequate answers to the surveys and 82 women 
were not included in the study because of not speaking and understanding in Turkish. In the end, a total of 223 women participated.

\section{The Edinburgh Postpartum Depression Scale (EPDS)}

The instrument which evaluates the postpartum depression status of women was first reported by Cox et al. [12], and the Turkish translation and validation were performed by Aydin et al. [11]. The scale has 10 self-reported questions, rated on a four-point Likert scale which are scored from 0 to 3 . The scale is scored between 0 and 30 and the cut-off point of 13 or higher scores are considered as the probable risk for the presence of postpartum depression.

\section{Maternal Attachment Inventory}

The Maternal Attachment Inventory (MAI) was described by Muller et al. [13] and its Turkish translation and validation were performed by Kavlak et al. [10]. It is a self-reported instrument with 26 questions which are scored from 1 (almost never) to 4 (almost always). The range of the scores is 26-104 and the higher scores show a higher maternal attachment.

\section{Statistical Analysis}

Statistical analysis was performed by SPSS (Statistical Package for the Social Sciences) 22 (SPSS Inc., Chicago, IL). The distribution of parameters was assessed by the KolmogorovSmirnov normality test. Descriptive analyses were given (using tables of frequencies for the categorical variables and) using medians and interquartile range for the non-normally

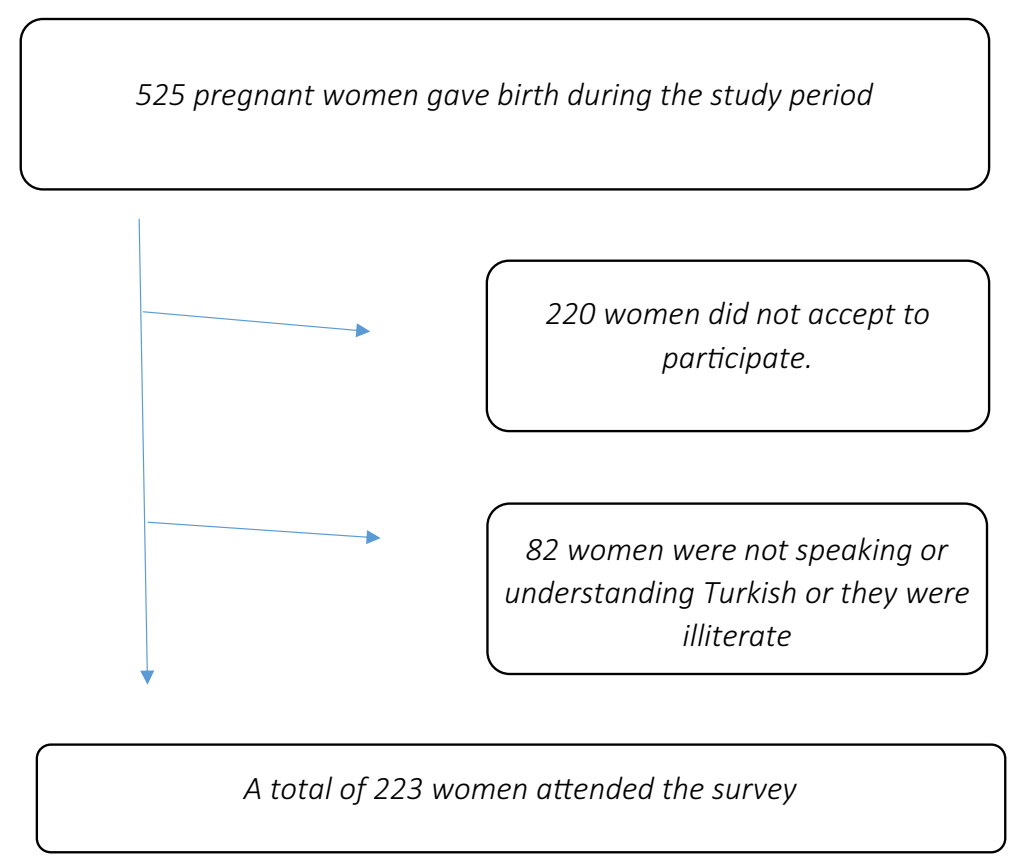

Fig. 1 The flowchart of women who attended the survey 
distributed and categorical variables and mean and standard deviation for the normally distributed variables. Mann Whitney-U test was performed for non-normal distributions. The comparison of categorical variables was performed by the chi-square test. $P$ values $<0.05$ were considered statistically significant.

For the power calculation, we accepted the post-partum depression prevalence as $7.8 \%$ for our population based on the previous literature, and the effect size as 0.3 . The total sample size of 205 was calculated by G-POWER 3.1.9.7 software with the alpha probability of 0.05 and a power of 0.95 [14].

\section{Results}

A total of 223 postpartum women within $48 \mathrm{~h}$ of birth participated in the study. The median (interquartile range) age of the women was 26 (9) years and the parity was 1 (2). The median gestational age was 39 (2) weeks and the median birth weight was 3290 (505) grams. One hundred and twenty-three women gave normal vaginal birth and $100(48.9 \%)$ women had cesarean section. From the newborns, 114 (51.1\%) were girls and 109 (48.9\%) boys (Table 1).

Regarding the educational status, $81(36.3 \%)$ women were illiterate or finished primary school, 119 (53.4\%) were high school graduates and $23(10.3 \%)$ were university graduates. Seventy-five (33.6\%) women were working while 148 (66.3\%) were housewives.

The median score obtained from the EPDS was 7 (7) and 33 (14.7\%) of the women were determined to have a risk for postpartum depression. The median scores of the EPDS inventory of depressive women were 15 (3).

The median MAI score of 223 women was 100 (26); and the MAI scores of women with depression were significantly lower than the controls [73 (39) vs. 101 (18) respectively, $\boldsymbol{p}<$ 0.001].

Table 1 Comparison of the main characteristics of women according to the presence of depressive symptoms

Depressive $(\operatorname{EPDS} \geq 13)(n=33) \quad$ No Depression $(\operatorname{EPDS}<13)(n=190) \quad \mathrm{P}$ Value

\begin{tabular}{lcrl}
\hline Education & & & \\
$\quad$ Illitarete/ primary school & $13(39.4 \%)$ & $68(35.8 \%)$ & 0.674 \\
High school & $18(54.5 \%)$ & $101(53.2 \%)$ & $<\mathbf{0 0 1}$ \\
University & $2(6.1 \%)$ & $21(11.1 \%)$ & $<\mathbf{0 0 1}$ \\
EPDS score & $15(3)$ & $6(5)$ & 0.338 \\
MAI score & $73(39)$ & $101(18)$ & 0.091 \\
Maternal age & $27(7)$ & $26(9)$ & 0.818 \\
Parity & $1(2)$ & $1(2)$ & $>0.999$ \\
Gestational week of birth & $38(1)$ & $39(2)$ & 0.209 \\
Delivery method & $18(54.4 \%)$ & $105(55.3 \%)$ & 0.346 \\
Vaginal birth & $15(45.5 \%)$ & $85(44.7 \%)$ & \\
Cesarean section & $3360(550)$ & $100(52.6 \%)$ & \\
Birthweight & $14(42.4 \%)$ & $90(47.4 \%)$ & \\
Fetal Gender & $19(57.6 \%)$ & & \\
Female & & & \\
Male & & & \\
\hline
\end{tabular}

- $\mathrm{P}$ values $<0.05$ were consşdered significant

- The values were expressed in median (interquartile range) or number (percentages) 


\section{Discussion}

The COVID-19 pandemic has a great impact worldwide in many areas. Not being only a healthcare issue, the quarantine period affects the psychosocial and economic life. Knowing that the post-partum period already has a risk of depression among women, the restricted living and giving birth in the restricted hospital conditions may end-up with decreased support for the vulnerable population and may affect the psychological status and the maternal-infant bonding [15]. In the current study, we evaluated the immediate post-partum women regarding the postpartum depression rates by using the EPDS and maternal-infant bonding by using MAI. 14.7\% of postpartum women had EPDS scores $>12$ and the median MAI score was 100 (26). We observed significantly lower maternal attachment scores in depressive mothers.

A previous report in April 2020 by Yassa et al. from Istanbul, Turkey evaluated the thirdtrimester pregnant women's attitude, concern and knowledge about the COVID-19 pandemic with a non-validated questionnaire and reported $87.2 \%$ of women complied with the selfquarantine rules, $52 \%$ felt vulnerable and $80 \%$ felt concerned, $35.5 \%$ constantly kept thinking about getting infected and $42 \%$ had concerns about their baby getting infected [16]. The aforementioned study leads us to investigate the post-partum depressive symptoms and the maternal bonding scores of the new mothers, who spent their last three-months under pandemic restrictions.

A study by Kokanali et al. performed a study on postpartum women in 2016-17 in the tertiary antenatal care center in Ankara, Turkey [14]. The study can be considered as a representative of the same population, as being performed in a tertiary care center of the same city, before the COVID pandemic. The study used the same questionnaires to evaluate the postpartum depression rates and maternal bonding in normal vaginal birth and cesarean section in 180 women. They have reported $7.8 \%$ of women who gave vaginal birth and $8.9 \%$ of women who had cesarean section had EPDS scores higher than 12 while the mean MAI scores were $100.47 \pm 5.05$ and $99.64 \pm 9.35$ respectively. Considering the approximately doubled rates of EPDS in our results in a similar population, we may put forward that, the postpartum depression risk in Turkish women during the COVID-19 pandemic was increased when compared with previous reports, while it was still lower than the studies reported from other countries. Besides, the median MAI score of our study was 100 (26), which was similar to the previous study before pandemic by Kokanali et al.

A report which evaluated the psychological impact of quarantine measures on immediate postpartum women in the north-eastern part of Italy, which was strongly affected by the pandemic reported a $26 \%$ rate of EPDS scores higher than 12. They compared the EPDS scores during the pandemic with the previous results of the control group who gave birth before the pandemic and they reported a significant worsening in depressive symptoms [5]. Furthermore, they evaluated the subscales of EPDS and reported significantly increased depression and anhedonia rates. Our study did not include a control group for comparison, but considering the variations in the health system, and the results of the study of Yassa et al. which indicated a $92.4 \%$ trust to the healthcare staff, an improvement in results could be expected [16]. Also, despite the high number of cases, an overload was not observed in Turkey and in the hospital where the study was performed, a triage for COVID-19 patients was performed and the suspected or diagnosed pregnant women were completely isolated in a different building for labor birth, while the COVID-19 negative presumed pregnant women gave birth and were hospitalized in single rooms while no relatives and even partners were let for visiting. Being isolated may affect the mood in a high-need period while the safety of the 
mother and the newborn was the priority. In other words, the feeling of being safe and isolated may be the cause of the better EPDS scores.

To the best of authors' knowledge, this is the first report on the psychological status of immediate post-partum women from Turkey, a country which had a three-months restriction and limited quarantine period while a high number of daily new cases were reported (https://covid19.saglik.gov.tr/). Only a few previous reports evaluated pregnant women. A preliminary on depression and anxiety symptoms of Turkish pregnant women were reported by Durankus and Aksu [17]. They evaluated 260 pregnant women and reported a $35.4 \%$ rate of women had EPDS scores higher than 13, which was more than twice of our reported rate. Their results showed a correlation with higher EPDS scores and years of education, maternal working status, and parity. In contradiction, lower rates of EPDS scores were found in our post-partum patients, also, no relationship was found between higher EPDS scores and maternal education status, maternal working status and parity. The aforementioned study was conducted in pregnant women, the study method used an online survey and the patients were from another city; which could be the factors for the great difference between the results.

The strength of our study is the face-to-face evaluation of the patients. The limitations of this study are the absence of a control group that was evaluated before the onset of pandemic and due to ongoing cases with a high incidence, it is uncertain when a control group will be available in near future. Also, a lack of any validated questionnaire for COVID-19 infection on psychological status would be a limitation for correlating the results with the pandemic. Therefore a preliminary data is reported and a comparison is made with previous reports from the same country and the only available data of EPDS in immediate postpartum women who gave birth during COVID-19 pandemic in a highly affected area in northwestern Italy [5].

\section{Conclusion}

In conclusion, providing appropriate isolation in hospitals for pregnant women may have a positive impact on the depressive symptoms of new mothers. Attention for the psychological status of pregnant and postpartum women may help for the improvement of psychosocial support. Evaluation of the factors that affect the psychological status of pregnant and postpartum women will lead the healthcare system to improve the implementations during the COVID-19 pandemic.

\section{Compliance with Ethical Standards}

The study was approved by the Ethics Committee of Ankara City Hospital in 04/06/2020 with the number 735 . All participants gave verbal and written informed consent. No funding was received for the study.

Conflict of Interest The authors declare no conflicts of interest.

\section{References}

1. Wang C, Horby PW, Hayden FG, Gao GF. A novel coronavirus outbreak of global health concern. Lancet. 2020;395(10223):470-3.

2. Turkish Ministry of Health, General Directorate of Public Health. COVID-19 (SARS-CoV-2 infection) Guideline, Scientific Committee Report 
3. Carlson A, Thung SF, Norwitz ER. H1N1 influenza in pregnancy: what all obstetric care providers ought to know. Rev Obstet Gynecol. 2009;2(3):139-45.

4. Jamieson DJ, Honein MA, Rasmussen SA, Williams JL, Swerdlow DL, Biggerstaff MS, et al. H1N1 2009 influenza virus infection during pregnancy in the USA. Lancet. 2009;374(9688):451-8. https://doi. org/10.1016/s0140-6736(09)61304-0.

5. Zanardo V, Manghina V, Giliberti L, Vettore M, Severino L, Straface G. Psychological impact of COVID19 quarantine measures in northeastern Italy on mothers in the immediate postpartum period. Int J Gynaecol Obstet. 2020;150(2):184-8. https://doi.org/10.1002/ijgo.13249.

6. Esterwood E, Saeed SA. Past epidemics, natural disasters, COVID19, and mental health: learning from history as we Deal with the present and prepare for the future. Psychiatry Q (2020) doi:https://doi. org/10.1007/s11126-020-09808-4.

7. Viaux S, Maurice P, Cohen D, Jouannic JM. Giving birth under lockdown during the COVID-19 epidemic. J Gynecol Obstet Hum Reprod. 2020;49(6):101785. https://doi.org/10.1016/j.jogoh.2020.101785.

8. Hermann A, Deligiannidis KM, Bergink V, Monk C, Fitelson EM, Robakis TK, Birndorf C Response to SARS-Covid-19-related visitor restrictions on labor and delivery wards in New York City. Arch Womens Ment Health (2020) doi:https://doi.org/10.1007/s00737-020-01030-2.

9. Abdoli A, Falahi S, Kenarkoohi A, Shams M, Mir H, Jahromi MAM The COVID-19 pandemic, psychological stress during pregnancy, and risk of neurodevelopmental disorders in offspring: a neglected consequence. J Psychosom Obstet Gynaecol: (2020) 1-2. doi:https://doi.org/10.1080/0167482 X.2020.1761321.

10. Kavlak O, Sirin A. The Turkish version of maternal attachment inventory. Journal of Human Sciences. 2009;6(1):188-202.

11. Aydin N, Inandi T, Yigit A, Nalan Sahin Hodoglugil N. Validation of the Turkish version of the Edinburgh postnatal depression scale among women within their first postpartum year. Soc Psychiatry Psychiatr Epidemiol. 2004;39(6):483-6. https://doi.org/10.1007/s00127-004-0770-4.

12. Cox J, Holden J, Sagovsky R. Edinburgh postnatal depression scale (EPDS). Br J Psychiatry. 1987;150: 782-6.

13. Müller ME. A questionnaire to measure mother-to-infant attachment. J Nurs Meas. 1994;2(2):129-41.

14. Kokanali D, Ayhan S, Devran A, Kokanali MK, Tasci Y. Effect of cesarean section on postpartum depression and maternal attachment. J Contemp Med. 2012;8(2):148-52.

15. Turkyilmaz E, Hesapcioglu ST. Adolescent Pregnancy's ongoing effects on the depression and anxiety scores in subsequent pregnancy. Gynecol Obstet Reprod med. 2019;25(3)):142-7. https://doi.org/10.21613 /gorm.2018.825.

16. Yassa M, Birol P, Yirmibes C, Usta C, Haydar A, Yassa A, Sandal K, Tekin AB, Tug N Near-term pregnant women's attitude toward, concern about and knowledge of the COVID-19 pandemic. J Matern Fetal Neonatal Med (2020):1-8. doi:https://doi.org/10.1080/14767058.2020.1763947.

17. Durankus F, Aksu E Effects of the COVID-19 pandemic on anxiety and depressive symptoms in pregnant women: a preliminary study. J Matern Fetal Neonatal Med (2020):1-7. doi:https://doi.org/10.1080 /14767058.2020.1763946.

Publisher's Note Springer Nature remains neutral with regard to jurisdictional claims in published maps and institutional affiliations.

Z. Asli Oskovi-Kaplan is an obstetrics and gynecology specialist with a high desire of scientific research especially in the area of maternal and neonatal medicine. She has a 5 years of experience as a specialist and she has contributed many scientific papers since residency.

Gul Nihal Buyuk is an obstetrics and gynecology specialist and has been actively working in the obstetrics care unit of a tertiary care maternal hospital which has been actively working also as a pandemic hospital. Dr. Buyuk has researches mainly in obstetrics are and maternal health.

A. Seval Ozgu-Erdinc is an associated professor of obstetrics and gynecology and has expertise in high risk pregnancies. The author has more than 50 scientific papers in the area and has been supervising many other researches. 
H. Levent Keskin in an associated professor of obstetrics and gynecology. Dr. Keskin is the head of the department of the obstetrics clinic and has been supervising many studies which are being conducted during pandemic.

Alper Ozbas is an obstetrics and gynecology resident and a promising future specialist.

Ozlem Moraloglu-Tekin is an obstetrics and gynecology professor and is the chief physician of the Ankara City Hospital- Obstetrics and Gynecology Hospital which is the tertiary referral antenatal hospital. She was previously the chief physician of a tertiary antenatal care center Etlik Zubeyde Hanim Women's Health Education and Research Hospital. She has been contributed many scientific researches in her area of expertise. 